\title{
Experimental and numerical study of phase mixing of an intense beam
}

\author{
D. Stratakis, ${ }^{*}$ R. A. Kishek, I. Haber, S. Bernal, M. Reiser, and P. G. O’Shea \\ Institute for Research in Electronics and Applied Physics, University of Maryland, College Park, Maryland 20742, USA
}

(Received 28 July 2008; published 1 June 2009)

\begin{abstract}
We study, experimentally and numerically, the relaxation of an initially nonuniform intense beam in an alternating-gradient transport line. A nonlinear distribution consisting of five interacting beamlets is created and tracked for longer than seven plasma periods with the help of tomographic phase-space mapping. Emittance growth is initially rapid, but slows down as the nonuniform distribution homogenizes in a few plasma periods. Both growth rates are found to depend on the beam current.
\end{abstract}

DOI: 10.1103/PhysRevSTAB.12.064201

PACS numbers: 29.27.-a, 52.59.Sa, 52.40.Mj, 52.35.- $\mathrm{g}$

Phase-space mixing of intense beams is a fundamental phenomenon that is of interest not only from the point of view of constructing high-current accelerators, but also because of the applicability of its basic physics to other systems of many particles interacting via long-range forces. Here we define "intense beams" as those containing a high density of particles in phase space, for which space-charge forces, or the mutual repulsion among particles, have a significant effect on particle dynamics within the beam. Examples include the beams within and near the injector of many recent and proposed accelerator applications, such as accelerator-driven neutron sources [1], higher-luminosity high-energy colliders [2], free electron lasers [3], energy recovery linacs [4], and heavy-ion inertial fusion drivers (HIF) [5]. For many such machines, damping is minimal; hence, structure born at the source can be transported far down the accelerator and can cause adverse effects downstream, such as emittance growth, halo, difficulty in focusing the beam, or coherent synchrotron radiation.

In addition to the importance of intense beam dynamics to accelerator physics, phase mixing in charged-particle beams has also been suggested [6-8] as a model to study collective dynamics in astrophysical phenomena such as galaxy interactions [9]. For example, we can learn whether phase mixing follows a power law or an exponential growth, the latter signifying a chaotic process. We can also compare mixing rates to the characteristic frequencies of the system, the galactic revolution time, or the plasma frequency in the case of beams.

To probe the mixing properties of charged-particle beams, we artificially construct a distribution consisting of five distinct but nearby beamlets in a quincunx pattern (Fig. 1). Experimentally this is performed by means of an aperture at the exit of a $10 \mathrm{keV}$ electron gun. Such a distribution is of interest because, from Poisson's equation, the radial nonlinearity of the self-fields is greatly enhanced.

*Present address: Brookhaven National Laboratory, Upton, NY 11973, USA.

diktys@bnl.gov
It is also of practical interest [10-12] because it relates to a class of transverse beam-combining lattice designs that have been proposed [13] to achieve high-current, low emittance beams in a cost-saving way for HIF applications. Studies at Berkeley [14-16] of the merging of four ion beams have shown, in fact, that such a concept is viable. In another example, transverse merging of many $(\approx 100)$ beams has been explored experimentally [17] and numerically [18] as an approach to generate high-current intense beams as it offers a number of potential advantages over a single-beam injector. We note however, that even though our experiments can be useful for multibeamlet guns exact correspondence is not the goal here due to the differences of the initial beam distribution function.

In addition to the above-mentioned HIF experiments, the propagation of five beamlets was previously studied in a low-energy solenoidal transport channel at the University

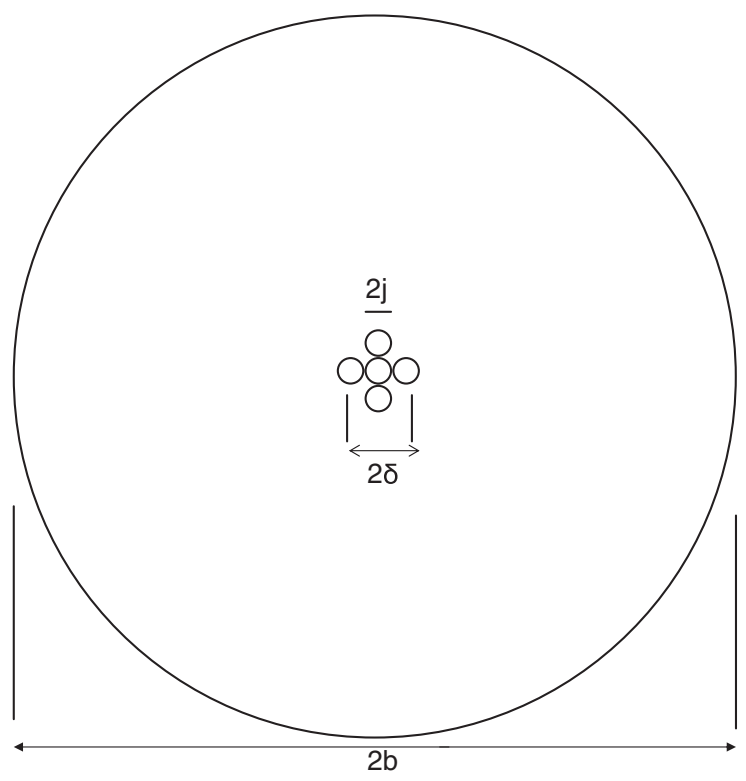

FIG. 1. Schematic layout of the UMER five-beamlet mask. Parameters $j$ and $b$ are the beamlet and pipe radius, respectively. For our experiment, $2 j=1.75 \mathrm{~mm}, 2 \delta=5.22 \mathrm{~mm}$, and $2 b=$ $50 \mathrm{~mm}$. Distances are to scale. 
of Maryland [19-21]. It was found that, though the individual beamlets quickly lost their identity, they reappeared in an image plane $1 / 2$ a betatron wavelength $(\sim 1 \mathrm{~m})$ from the source. The failure of the beamlets to reappear in subsequent image planes suggested a collisionless process that caused the particles to lose memory of their initial conditions. Particle-in-cell (PIC) simulations successfully reproduced those results in detail $[21,22]$. Further simulations employing an initial five-beamlet configuration in a constant uniform solenoidal transport channel showed that clumps of particles initially localized in phase space grew exponentially to fill their entire accessible phase-space regions, suggesting chaotic mixing $[7,23]$.

However, the previous studies left several questions unanswered. First, those experiments were limited to a propagation distance to about $5 \mathrm{~m}$. Will the distribution continue to evolve beyond that distance? Second, how will the alternating gradient of a strong-focusing lattice, such as used by most accelerators, affect the results, if any? Third, the old experiments measured the beam only in configuration space, and any detailed knowledge of phase space was inferred from simulation. What can we learn if we can apply a direct measurement of phase space? This work, which attempts to address these questions, is aided by several developments: the existence of the University of Maryland Electron Ring (UMER) [7], which possesses both a strong-focusing lattice and a much longer propagation distance, and the development of a novel tomographic technique that provides a high-fidelity mapping of phase space from experimental data [24-29].

We examine here both experimentally and numerically the evolution and mixing mechanisms as well as time scales of a multibeamlet charged-particle distribution in UMER, relying on data in both configuration and phase space. We found that the beamlet distribution initially undergoes rapid mixing accompanied by a quick emittance growth, followed by a slower homogenization taking place within a few plasma periods. Simulations elucidate the dependence of the two growth rates on the relative strength of the space-charge forces.

Tomography is a technique [27] by which an $N$-dimensional object is reconstructed from projections in $N-1$ dimensions, e.g., reconstruction of a 3D object from 2D images obtained at various angles. For particle beams, tomographic techniques can be applied to recover the phase-space distribution from a large number of projections (50-200) onto configuration space from multiple angles [24,25], where quadrupole or solenoid lenses are used to rotate the beam in phase space while collecting photographs on a fluorescent screen downstream. Thus, the tomographically constructed phase spaces contain a high density of information.

The tomography algorithm is adjusted to account for the beam space charge [24-26] assuming linear space-charge forces corresponding to a uniform beam density. It is worth noting that the tomographic measurements described herein employ beams that are far more intense than those described in any other tomographic measurement to date. Given the large deviations of the five-beamlet distribution from spatial uniformity and the resulting nonlinear selfforces, the accuracy of the tomographic reconstruction is in question. To verify the technique, we therefore simulated the process using 200 PIC simulations to generate the projections, and compared the phase space obtained directly from simulation to that reconstructed from the data. Then, we computed the $4 \times$ rms emittances from those phase spaces. We found that the systematic errors when using tomography to measure the emittance of a spacecharge dominated beam fit an empirical expression that follows the formula error $(\%)=3+9.8 \chi^{2.7}$, where $\chi$ is a dimensionless ratio that, as we will show later, is related to the beam space-charge intensity. In applying this to the five-beamlet case which we use in our experiment $(\chi=$ 0.70 ), this predicts $7 \%$. Hence, when we measure the beam emittance by tomography we always make a $7 \%$ correction to account for this systematic error.

Our experiment was carried out on UMER, where a wheel near the anode plane of the thermionic gun contains apertures which are used to mask the initial beam cross section, including the quincunx aperture described earlier (Fig. 1). The mask is followed by a $1.4 \mathrm{~m}$-injection line containing one solenoid, six matching quadrupoles, and one Bergoz fast current transformer (FCT). The UMER ring consists of 18 sections each containing four quadrupoles and two dipoles, distributed over a $11.52 \mathrm{~m}$ circumference. With a zero-current phase advance, $\sigma_{0}$, of $76^{0}$ and lattice periodicity, $P$, of $0.32 \mathrm{~m}$, UMER beams have a zerocurrent betatron oscillation wavelength, $\lambda_{\beta 0}$, equal to $1.51 \mathrm{~m}$. All but four sections house diagnostic chambers each containing a fluorescent screen to image the transverse beam distribution.

The beam emerging from the five-beamlet mask has a current of $28 \mathrm{~mA}$ measured by the Bergoz FCT coil, and an rms normalized emittance, $\varepsilon_{i, n}$, of $4 \mu \mathrm{m}$ [7], measured by tomography and verified with a pinhole scan. Note that the beam is flattop meaning that the line charge density is constant throughout the pulse. The beam parameters correspond to a generalized perveance, $K$, of $4.2 \times 10^{-4}$ and an intensity parameter $\chi=0.70$, which places our beam in the space-charge-dominated regime. The dimensionless intensity parameter, $\chi$, is defined as the ratio of the spacecharge force to the external focusing force at the beam edge, in the smooth approximation [30], which in terms of measured beam parameters reduces to [24]: $\chi=$ $\left(1+8 \beta \gamma I_{0} a^{-2} \varepsilon_{i, n}^{2} / I\right)^{-1}$ where $\beta, \gamma$ are the relativistic velocity and energy factors, respectively, and $a$ is the matched $2 \times$ rms beam radius. For electrons, $I_{0}$ is equal to $17 \mathrm{kA}$. Since space charge is repulsive for beams of the same sign of charge, it lowers the betatron oscillation frequency resulting in an increase in betatron wavelength 
$\lambda_{\beta}>\lambda_{\beta 0}$. The ratio of these wavelengths is related to the intensity parameter by $\lambda_{\beta} / \lambda_{\beta 0}=(1-\chi)^{-1 / 2}$. Beams with space charge can support waves which are characterized by a plasma wavelength $\lambda_{p}$, connected to the intensity parameter by $\lambda_{p} / \lambda_{\beta 0}=(2 \chi)^{-1 / 2}$. For our beam $\lambda_{\beta}=$ $2.75 \mathrm{~m}$ and $\lambda_{p}=1.3 \mathrm{~m}$.

Figure 2(a) (left) shows a phosphor screen image of the UMER beam distribution in the injector line at an approximate distance $\approx \lambda_{\beta} / 4$ downstream from the source. Figure 2(a) (right) is the tomographically reconstructed $x x^{\prime}$ phase space at the same location. Even though the original beamlet pattern is only faintly visible in configuration space, the beamlets are more visibly separated in phase space. The persistence of the pattern in phase space means that memory of the initial distribution is retained. Note that focusing the beam implies a rotation of the phase space, so the pattern naturally alternates between appearances in configuration and in velocity space. An erasure of this phase-space pattern corresponds to a mixing of the distribution, which as we shall see happens further downstream.

Self-consistent PIC simulations have been performed with the $2 \frac{1}{2} \mathrm{D}$ version of WARP [31], which advances particles in a transverse slice under the influence of external forces and self-consistent self-fields. We use a $512 \times$ 512 spatial grid, a step size of $z=2 \mathrm{~mm}$ in the axial direction of the beam propagation and 640000 particles. Note that running a large number of test simulations with more particles or higher resolution resulted in no perceptible difference in the final result. Figure 2(b) illustrates the
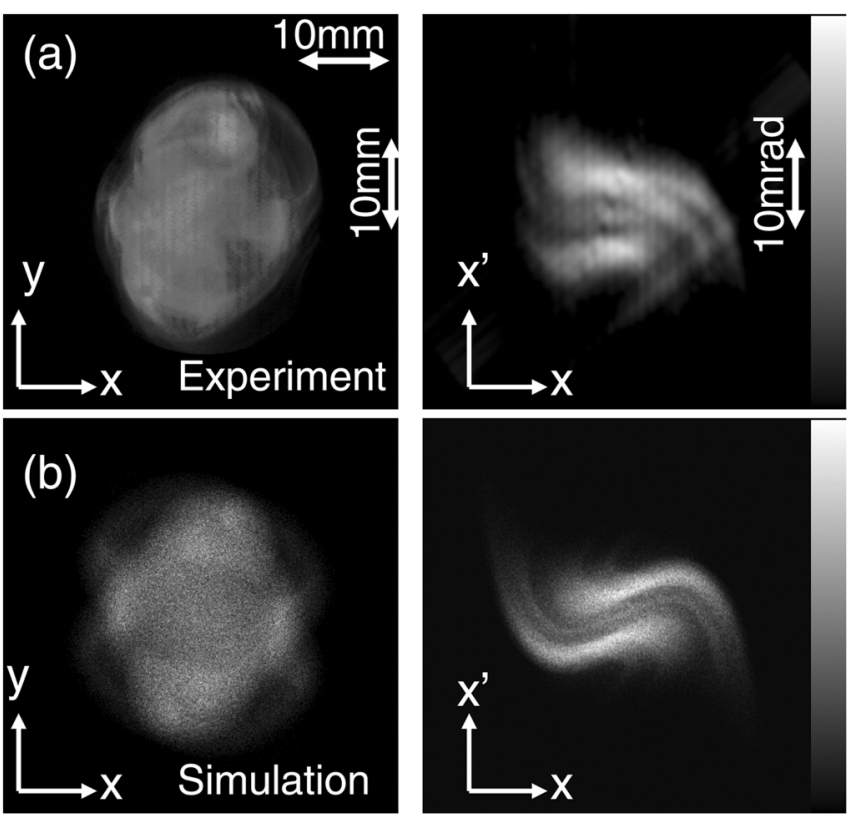

FIG. 2. Merger of the five beamlets at a distance $\approx 0.6 \lambda_{p}$ from the source. Configuration space (left) and $x x^{\prime}$ phase space (right) from (a) experiment and (b) WARP simulation. The measured emittance in terms of the initial $4 \times \mathrm{rms}$ emittance, $\varepsilon_{i}$, is $1.05 \varepsilon_{i}$. configuration space (left) and phase space (right) obtained directly by the WARP simulation, which shows the beamlets merged in configuration space and more distinct in phase space in agreement with the experiment.

Figure 3 (left) shows several fluorescent screen pictures taken in the experiment labeled with the distance from the UMER aperture (in units of plasma periods, $\lambda_{p}$ ). Figure 3 (right) shows the $x x^{\prime}$ phase-space distribution measured by tomography at the same locations. The images indicate that the beam core retains an intricate dynamical structure over the first five plasma periods and then becomes more homogenized in both configuration and phase space. It is worth noting that repeating the experiment for a beam with similar parameters that is initially uniform we also see structures, resulting from the imbalance of forces at the beam edge [32]. However, their evolution and mixing rates are different from the fast and violent mixing seen with the five beamlet in the sense that they require longer time scales [33]. Given that the characteristic time scale for interparticle collisions in UMER corresponds to 1000 plasma periods [7], this fast relaxation to equilibrium in the five-beamlet case cannot be associated with interparticle collisions but is rather generated by the influence of the nonlinear space-charge forces. We note here the appearance of a halo, approximately 2.4 plasma periods from

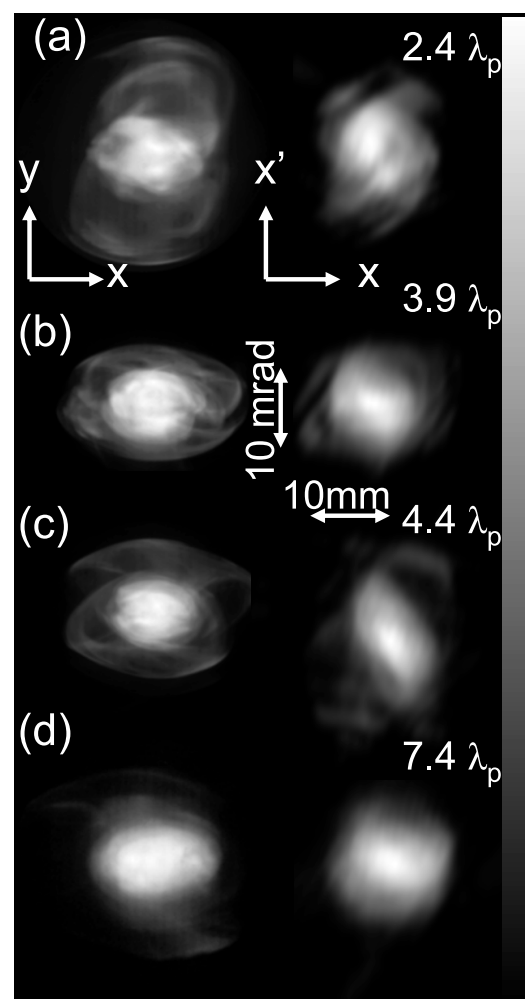

FIG. 3. Beam evolution at different locations inside the UMER ring in configuration space (left) and $x x^{\prime}$ phase space (right). The measured emittances in terms of the initial $4 \times$ rms emittance, $\varepsilon_{i}$, are (a) $1.09 \varepsilon_{i}$, (b) $1.08 \varepsilon_{i}$, (c) $1.11 \varepsilon_{i}$, and (d) $1.10 \varepsilon_{i}$. Those values are also shown with red squares in Fig. 4. 
the aperture [Fig. 3(a)], which persists for the remaining length of the focusing channel. WARP simulations indicated that the halo likely is a result of a small beam mismatch [30] and imperfections of the gun geometry [34].

According to the free energy theory [30], a nonuniform beam has higher field energy than the equivalent uniform beam and the particle distribution will eventually become uniform $[19,35]$. Such a configuration has been shown to be a minimum of electrostatic field energy [36,37]. Emittance growth is expected to result from the conversion of the excess field energy of a nonuniform beam into transverse kinetic energy. It was shown numerically [35] and analytically $[36,38]$ that most growth will occur within a fraction of a plasma period. For a matched beam, the emittance growth predicted by that model and its dependence on the initial beam position and size has been calculated analytically [16] and is given by

$$
\varepsilon_{f} / \varepsilon_{i}=\left(1+K a^{2} U / 2 \varepsilon_{i}^{2} w_{0}\right)^{1 / 2},
$$

where $\varepsilon_{i}$ and $\varepsilon_{f}$ are the initial and final $4 \times$ rms emittances, and $U / w_{0}$ is a dimensionless quantity [19] that depends on the shape of the initial nonuniform distribution. Earlier experiments in Maryland [19-21] and Berkeley [16,39] have verified Eq. (1). For our experimental parameters $U / w_{0}$ is equal to -0.27 and the radius, $a$, is equal to $5.98 \mathrm{~mm}$. Therefore, the above model predicts an emittance increase by a factor of 1.1, which is close to the growth measured by tomography from the phase space in Fig. 2 .

Figure 4(a), obtained from a WARP simulation, shows the emittance evolution over a long distance for a beam with the experimental parameters. The initial beam conditions are chosen to be matched solutions of the rms envelope equations [30]. The red squares correspond to experimental data from the phase spaces of Figs. 2 and 3. Three distinct phases in the evolution of the beam cross section have been observed in the simulations with relatively sharp transitions between phases. The location of those transition points is denoted by vertical arrows in Fig. 4(a). The first phase is characterized by a clearly observable presence of the images of the initial fivebeamlet pattern. In this phase the images of the initial configuration are found to repeatedly reappear with an approximate half betatron period. Since this phase is also characterized by a rapid growth in the emittance, we call this the fast growth phase. In the second phase, the beam cross section is still seen to be evolving toward steady state, but the initial five-beamlet pattern is not readily apparent. The emittance generally shows a high level of fluctuations in this phase, but some moderate growth is also seen. We therefore call this the phase of slow growth. The third, no growth phase, is a near steady state where the beam becomes completely mixed, i.e., we cannot distinguish any patterns within the beam distribution, nor is any emittance growth observed. Note that, compared with the initial steep increase, the PIC simulation indicates a relatively slow
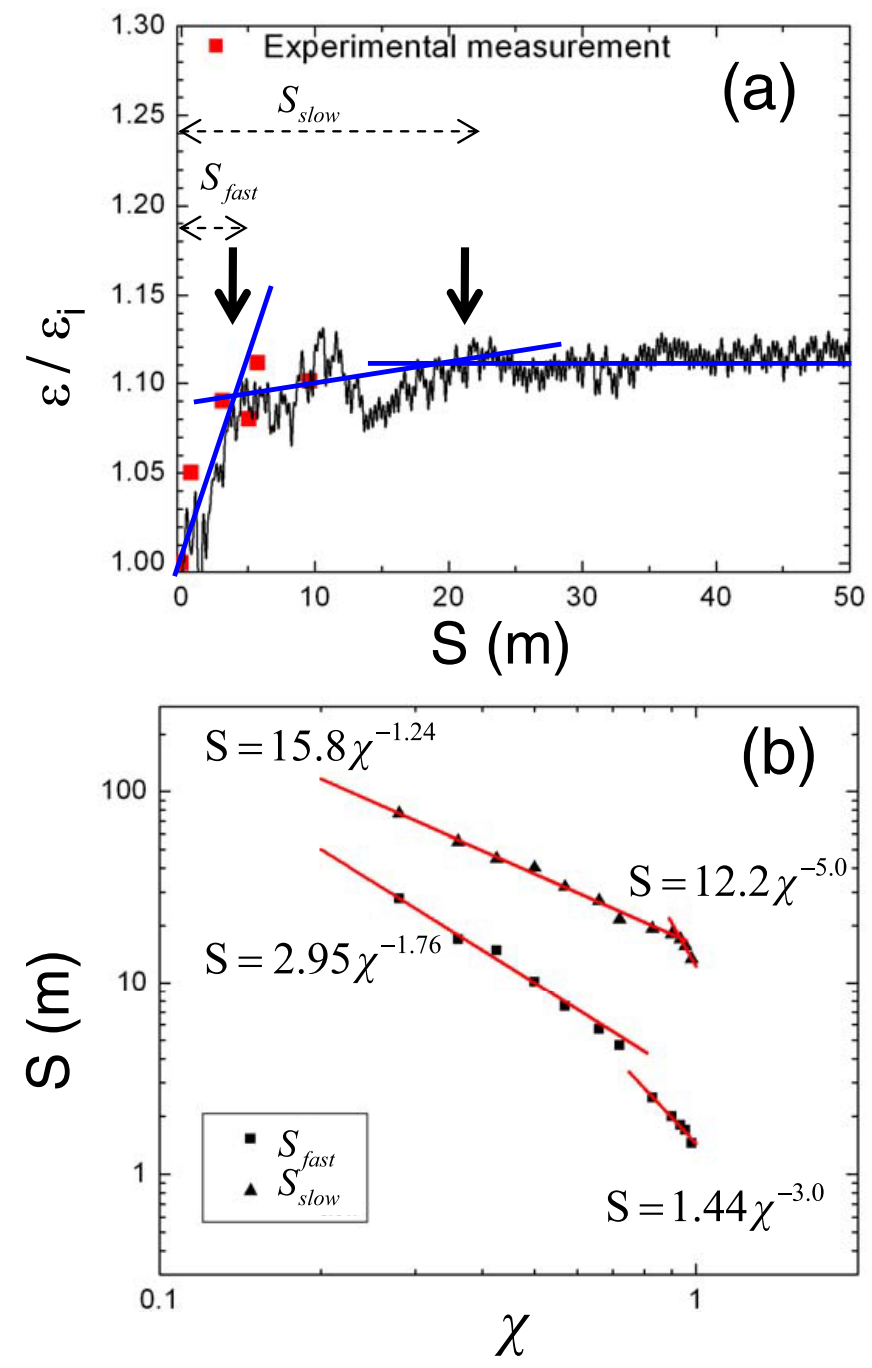

FIG. 4. (Color) Evolution of phase mixing from simulation: (a) emittance growth rates for beam parameters as in the experiment $(\chi=0.7)$. The red squares are experimental data. (b) Dependence of growth rates with the beam intensity $\chi$ in logarithmic scale. The mathematical expressions are obtained by fitting the data (red lines).

emittance growth after about three plasma periods $(S>$ $3 \mathrm{~m}$ ) and likewise, the experimental data show less emittance fluctuations after the first 2-3 plasma periods.

It is also interesting to examine the parametric dependence of the emittance growth. We define a characteristic distance for the change of the growth rate by $S_{\text {fast }}$ and define another distance where the emittance approaches its final value by $S_{\text {slow }}$ [Fig. 4(a)]. Then, in Fig. 4(b) we systematically vary in our simulation the beam current to explore the dependence of those transition distances to the ratio related to the space-charge intensity, $\chi$. The red lines are a fit to the data, with mathematical expressions shown in Fig. 4(b). Note that for all cases the initial beam conditions are chosen to be matched solutions of the rms envelope equations. As space-charge intensity increases, the beam is seen to progress faster toward relaxation 
suggesting that stronger space-charge enhances the mixing rate. Note that the opposite result was found for a spacecharge dominating beam starting with a semi-Gaussian (S-G) distribution [32], and this was because an equilibrium distribution converges to a $S-G$ in the space-charge limit. Thus, not only the intensity but also the beam initial distribution determines its subsequent evolution.

In this study we examined experimentally and numerically phase-space mixing of an intense beam. We found that a distribution with a large initial nonuniformity will first rapidly mix with a quick emittance growth, followed by a slower homogenization taking place within a few plasma periods. Noting that the mixing rates and their dependence on intensity are partially a function of the initial distribution, we suggest for a future study to increase or reduce the number of beamlets in the simulation in order to further explore this dependence. Additionally, it will be interesting to look more closely at the observed beam halo, which in the case of UMER had multiple sources including an imperfection in the gun geometry that has since been corrected.

\section{ACKNOWLEDGMENTS}

We thank M. Walter, S. M. Lund, B. Beaudoin, E. Voorhies, and C. Papadopoulos. This work is supported by the U.S. Department of Energy High Energy Physics and Fusion Energy Science, and by the U.S. Department of Defense Office of Naval Research and Joint Technology Office.

[1] J. Wei et al., in Proceedings of the 20th Particle Accelerator Conference, Portland, OR, 2003 (IEEE, New York, 2003), p. 571.

[2] R. B. Palmer et al., in Proceedings of the 2007 Particle Accelerator Conference, Albuquerque, NM (IEEE, New York, 2007), p. 3193.

[3] C. A. Brau, Free Electron Lasers (Academic Press, San Diego, 1990).

[4] G. H. Hoffstaetter and Y.H. Lau, Phys. Rev. ST Accel. Beams 11, 070701 (2008).

[5] S. S. Yu et al., Nucl. Fusion 47, 721 (2007).

[6] H.E. Kandrup et al., Ann. N.Y. Acad. Sci. 1045, 12 (2005).

[7] R. A. Kishek et al., Ann. N.Y. Acad. Sci. 1045, 45 (2005).

[8] C. L. Bohn and I. V. Sideris, Phys. Rev. ST Accel. Beams 6, 034203 (2003).

[9] C. Nipoti, P. Londrillo, and L. Ciotti, Mon. Not. R. Astron. Soc. 344, 748 (2003).

[10] R. Noble and J. Spencer, in Proceedings of the 2007 Particle Accelerator Conference, Albuquerque, NM (Ref. [2]), p. 3112.
[11] G. M. Gammel, A. W. Maschke, and R. M. Mobley, Rev. Sci. Instrum. 52, 971 (1981).

[12] M. Rihaoui, C. L. Bohn, P. Piot, and J. G. Power, in Proceedings of the 2007 Particle Accelerator Conference, Albuquerque, NM (Ref. [2]), p. 4027.

[13] C. M. Celata, AIP Conf. Proc. 152, 278 (1986).

[14] P. Seidl et al., Phys. Rev. ST Accel. Beams 6, 090101 (2003).

[15] C. M. Celata et al., Fusion Eng. Des. 32-33, 219 (1996).

[16] C. M. Celata, A. Faltens, D. Judd, L. Smith, and M. G. Tiefenback, Proceedings of the Particle Accelerator Conference, Washington, DC, 1987 (IEEE, Piscataway, NJ, 1987), p. 1167.

[17] J. W. Kwan et al., Nucl. Instrum. Methods Phys. Res., Sect. A 544, 134 (2005).

[18] D. P. Grote, J. W. Kwan, and G. A. Westenskow, Nucl. Instrum. Methods Phys. Res., Sect. A 577, 58 (2007).

[19] M. Reiser et al., Phys. Rev. Lett. 61, 2933 (1988).

[20] D. Kehne, Ph.D. dissertation, University of Maryland, College Park, 1992.

[21] I. Haber, D. Kehne, M. Reiser, and H. Rudd, Phys. Rev. A 44, 5194 (1991).

[22] R. A. Kishek et al., in Proceedings of the Particle Accelerator Conference, Chicago, IL, 2001 (IEEE, New York, 2001), p. 151.

[23] C. L. Bohn, I. V. Sideris, H. E. Kandrup, and R. A. Kishek, Proceedings of the 21st International Linac Conference, Gyeongju, South Korea, 2002 (Pohang Accelerator Laboratory, Pohang, Korea, 2003), p. 389.

[24] D. Stratakis et al., Phys. Rev. ST Accel. Beams 9, 112801 (2006).

[25] D. Stratakis, K. Tian, R. A. Kishek, I. Haber, M. Reiser, and P. G. O'Shea, Phys. Plasmas 14, 120703 (2007).

[26] D. Stratakis et al., Phys. Rev. ST Accel. Beams 12, 020101 (2009).

[27] J. Radon, Math. Phys. Klasse 69, 262 (1917).

[28] F. Zhou et al., Phys. Rev. ST Accel. Beams 9, 114201 (2006).

[29] V. Yakimenko, M. Babzien, I. Ben-Zvi, R. Malone, and X.-J Wang, Phys. Rev. ST Accel. Beams 6, 122801 (2003).

[30] M. Reiser, Theory and Design of Charged Particle Beams (John Wiley \& Sons, New York, 1994).

[31] A. Friedman, Nucl. Instrum. Methods Phys. Res., Sect. A 544, 160 (2005).

[32] R. A. Kishek et al., Phys. Rev. Lett. 85, 4514 (2000).

[33] D. Stratakis, Ph.D. dissertation, University of Maryland, College Park, 2008, Chap. 4.

[34] I. Haber et al., in Proceedings of the 2007 Particle Accelerator Conference, Albuquerque, NM (Ref. [2]), p. 3564.

[35] T. P. Wangler, K. R. Crandall, R. S. Mills, and M. Reiser, IEEE Trans. Nucl. Sci. 32, 2196 (1985).

[36] O. A. Anderson, Part. Accel. 21, 197 (1987).

[37] I. Hofmann and J. Struckmeier, GSI Report GSI 86-11, 1986.

[38] J. M. Dawson, Phys. Rev. 113, 383 (1959).

[39] M. G. Tiefenback and D. Keefe, IEEE Trans. Nucl. Sci. 32, 2483 (1985). 